\title{
Cutaneous ileocystostomy (a bladder chimney) for the treatment of severe neurogenic vesical dysfunction
}

\author{
DA Rivas ${ }^{1}$, S Karasick ${ }^{2}$ and MB Chancellor ${ }^{1}$ \\ ${ }^{1}$ Assistant Professor of Urology, Department of Urology, ${ }^{2}$ Clinical Professor of Radiology, Department of \\ Radiology, Jefferson Medical College, Philadelphia, PA 19107, USA
}

\begin{abstract}
The aim of this study was to investigate the efficacy and morbidity of cutaneous ileocystostomy, as an alternative to cystectomy and ileal conduit urinary diversion, for patients with end-stage neurogenic vesical dysfunction. Three male and eight female patients, mean age 41 years (range 28-59), with a mean duration of a neuropathic bladder of 8 years (range 4-17 years) underwent evaluation for ileocystostomy urinary diversion. Indications for the procedure included a bladder capacity $\leqslant 200 \mathrm{ml}$ (10 patients), recurrent febrile urinary tract infection (nine patients), and urinary incontinence despite an indwelling urethral catheter (all eight women). Each was felt to be a poor candidate for, or refused, continent urinary diversion or bladder augmentation cystoplasty. All eight females required concomitant pubovaginal sling urethral compression to eliminate urinary leakage from a patulous, non-functional urethra. Two patients required bilateral ureteral reimplantation for grade III-IV/V reflux. Effective low-pressure urinary stomal drainage was achieved without the need for chronic catheterization in all of the patients with a mean duration of follow-up of 24 months (range 6-60 months). No patient has developed pyelonephritis since the procedure. Urethral urinary leakage was eliminated in all of the female patients, whilst vesicoureteral reflux resolved in those with reflux preoperatively.
\end{abstract}

Keywords: neuropathic bladder; stress urinary incontinence; spinal cord injury; urethra; bladder; urodynamics

\section{Introduction}

Patients with advanced neuropathic vesical dysfunction are often managed by indwelling urinary catheter drainage. The misconception is prevalent that women tolerate a chronically indwelling catheter with less morbidity than men. ${ }^{1}$ Chronic catheterization, however, is associated with significant complications, including the development of neoplasia, febrile urinary tract infection, calculus formation, impaired renal function, diminished bladder capacity and compliance, and destruction of the continence mechanism..$^{2-5}$

One of the most challenging problems in the management of patients with neurogenic vesical dysfunction is the establishment of low-pressure urinary drainage without the use of an indwelling urethral or suprapubic catheter. Such drainage may be accomplished by a cutaneous ileocystostomy, using a small section of terminal ileum to act as a conduit between the urinary bladder and the anterior abdominal wall; an ileal 'bladder chimney'. This procedure was reported in 1957 by Cordonnier, ${ }^{6}$ but subsequently attention focused on augmentation cystoplasty versus formal urinary diversion.

Several distinct advantages, however, may be attributed to the bladder chimney operation. The procedure avoids manipulation of the ureters and thereby eliminates the problems associated with uretero- intestinal anastomoses such as reflux, stenosis, devascularization, urinary extravasation, or complete anastomotic disruption. Urinary collection is achieved with a standard urostomy drainage bag, without the need for an invasive foreign body which would predispose to chronic infection, neoplasia, or calculus formation. In addition, the gradual destruction of the bladder associated with indwelling catheters is obviated by this effective drainage procedure.

It must be noted that those afflicted with severe neurological impairment are unendingly hopeful of full recovery. The bladder chimney becomes an extremely attractive method for urinary tract management to those forever-hopeful individuals because it is a potentially reversible operation. The ileal chimney aspect could be amputated and the bladder easily reconstructed if the patient were spontaneously to regain bladder function, or techniques, such as functional electrical stimulation, are developed to restore normal detrusor function.

In those patients who have already suffered the ill effect of sphincteric insufficiency as a result of an indwelling catheter, pubovaginal sling urethral compression affords perineal dryness while urinary drainage is maintained through the bladder chimney. ${ }^{7-9}$ This manuscript represents the recent experience with the creation of a cutaneous ileocystostomy, a 
bladder chimney, in patients with severe neurogenic vesical dysfunction at the Thomas Jefferson University Hospital.

\section{Materials and methods}

Over the past 5 years three male and eight female patients underwent cutaneous ileocystostomy urinary diversion as the treatment for severe neurogenic vesical dysfunction. Indications for the procedure included a bladder capacity $\leqslant 200 \mathrm{ml}$ (10 patients), recurrent febrile urinary tract infection (nine patients), urinary incontinence despite an indwelling urethral catheter (all eight women), and vesicoureteral reflux (four renal units in two patients). The age of the patients, their underlying neurological disease, and the duration of indwelling catheterization are listed in Table 1.

Ten patients had been managed chronically with an indwelling urethral catheter and the remaining patient with a suprapubic tube. Eight patients, either with incomplete SCI or another etiology for bladder dysfunction which did not impair pain sensation, complained of chronic pelvic pain. Six patients suffered episodes of urinary sepsis during the 12 months prior to surgery which required hospitalization. Two cervical spinal cord injury patients suffered subjective symptoms of autonomic dysreflexia which included headache, diaphoresis, and facial flushing. Two patients who had demonstrated grade III-IV/V vesicoureteral reflux at cystography underwent simultaneous ureteral reimplantation. Two patients developed sacral decubitus ulceration as a consequence of their urinary incontinence and required additional myocutaneous surgery.

Preoperative evaluation included a complete videourodynamic study, cystoscopy, and upper urinary tract imaging with either intravenous pyelography, renal ultrasonography or a radioisotope renal scan. A history of bladder carcinoma or urine cytology consistent with malignancy were contraindications for the procedure.

All eight female patients required pubovaginal sling urethral compression to achieve functional bladder neck closure as indwelling catheterization had destroyed continence function. Each patient required an intact bladder neck and at least $1 \mathrm{~cm}$ of proximal urethra in order to effectively place the compressing pubovaginal sling.

\section{Technique of cutaneous ileocystostomy}

Adequate exposure was obtained through a midline or transverse abdominal incision. An ileal segment was isolated using a segment of ileum taken approximately $12-20 \mathrm{~cm}$ proximal to the ileocecal valve. The length of the segment required to bridge the gap between the bladder dome and abdominal surface was dependent upon the size of the individual, although $15 \mathrm{~cm}$ was adequate in most cases. Anastomosis between the ileal segment and the bladder was accomplished by a running single layer of 2-0 Vicryl $^{\mathrm{R}}$ (Ethicon, Somerville, New Jersey). The distal end of the ileal segment was

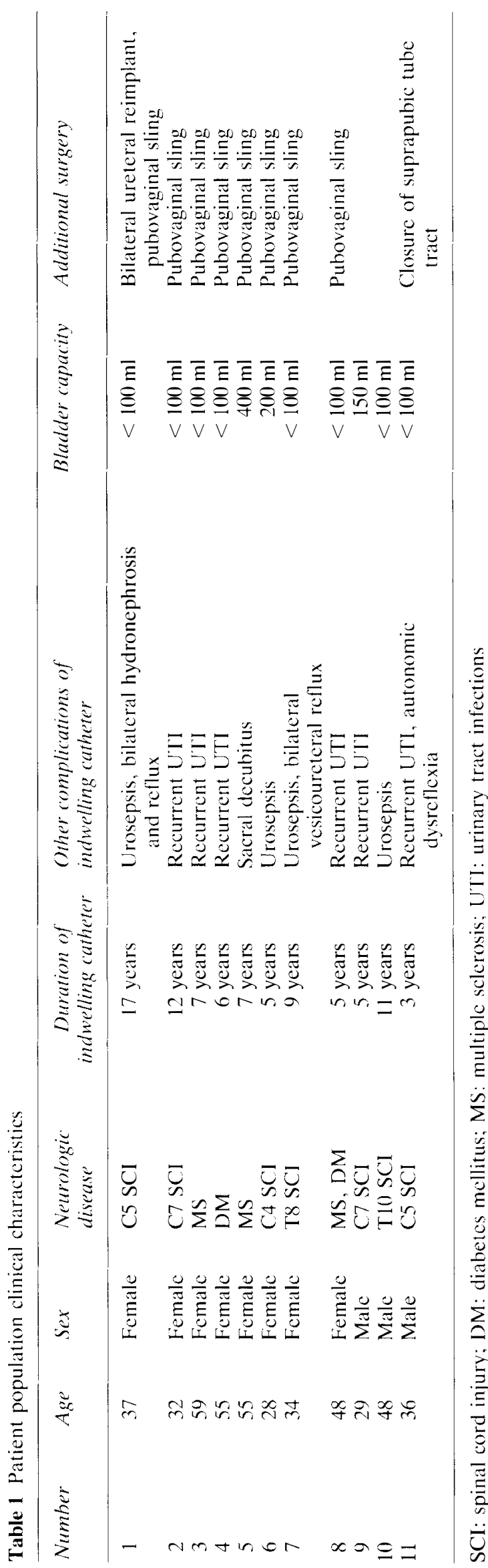


brought out to the abdominal wall at a point generally used for an ileal loop conduit stoma (Figure 1).

There are two important points in this operation. The first is to use as short a segment of ileum as

a

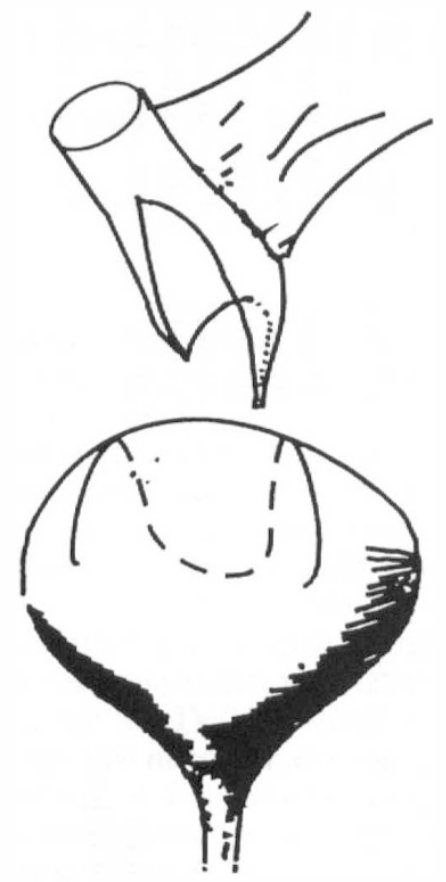

b

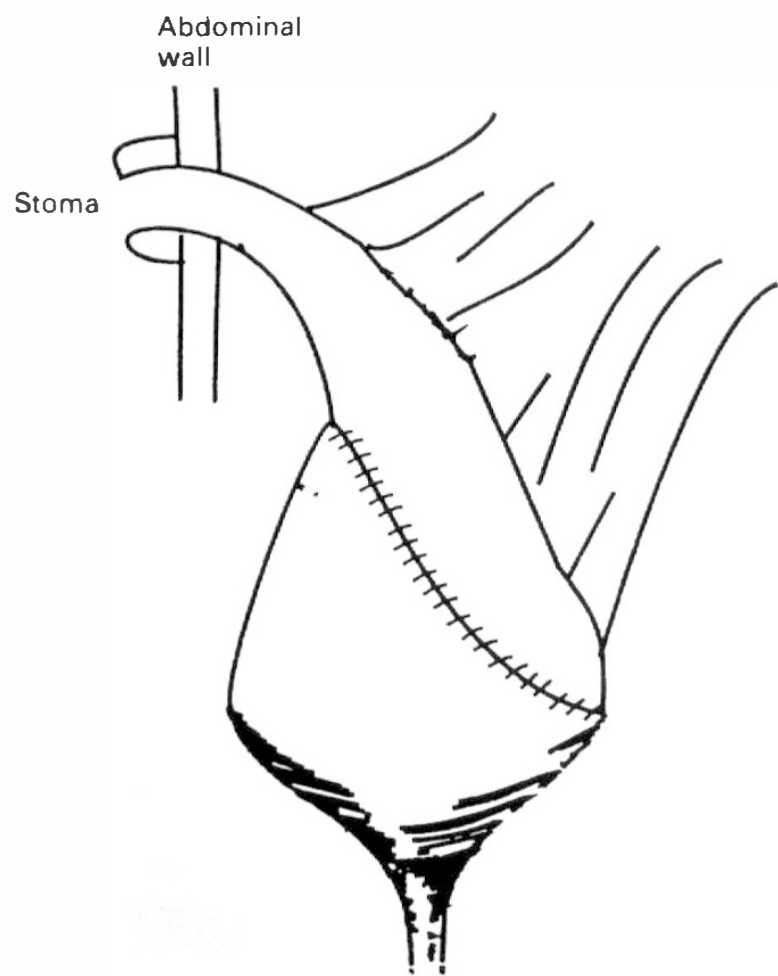

Figure 1 (a) Bladder with boari flap and isolated ileal segment with partial detubularization. (b) Lateral view of anastomosis of bladder and ileal chimney with cutaneous urostomy possible. There should be a wide, straight route from the bladder to the stoma. Redundancy of the bowel segment will inhibit urinary flow through the conduit and potentially contribute to electrolyte disturbance. The second is to avoid a circular anastomosis between the ileum and bladder. This may contract concentrically, producing an hourglass deformity and urinary stasis postoperatively. We used a modified Boari flap on both the bladder dome and proximal ileal segment to create a spatulated anastomosis configuration. Drainage was assured via a red rubber catheter placed through the urostomy into the bladder. Multiple side holes were placed in this catheter to avoid plugging with intestinal mucus. A closed-system perivesical drain was placed through a stab incision in order to avoid urinoma formation. Nasogastric tube suction was used continuously in all patients until there was resolution of postoperative ileus. When indicated, oral anticholinergic medications were initiated empirically to decrease detrusor spasticity and thereby assist healing of the ileovesical anastomosis.

An ileocystogram was performed approximately 1 week postoperatively to demonstrate an intact anastomosis without extravasation (Figure 2). After this,

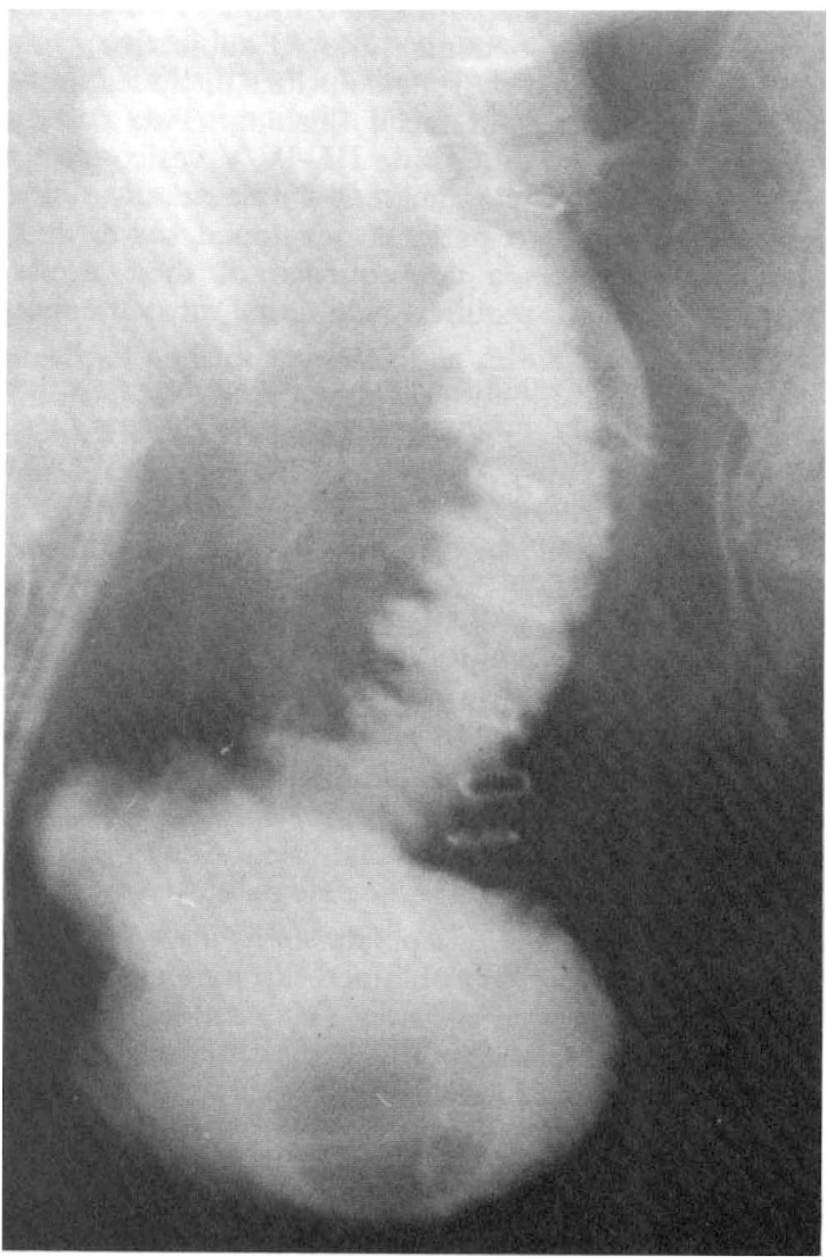

Figure 2 Cystogram of ileocystoplasty with cutaneous urostomy 'bladder chimney' status post pubovaginal sling 
removal of the drain and the initiation of dependent bladder chimney drainage was permitted.

\section{Results}

The ileocystostomy procedure effectively established low-pressure urinary drainage in all patients (Figure 3). Postoperative cystography demonstrated flow of urine from the bladder draining from the cutaneous urostomy without extravasation in all cases. The three men maintained reflex erections postoperatively.

Long term follow-up ranged from 6 to 60 months (mean 24 months) for the 11 patients. Cystography demonstrated resolution of reflux in the two patients with preexisting vesicoureteral reflux. One patient exhibited resolution of vesicoureteral reflux after ureteral reimplantation. In another patient, however, reflux resolved spontaneously after low-pressure urinary drainage was established with the bladder chimney procedure. All women were completely free from urinary leakage per urethra after pubovaginal sling functional urethral closure. The pubovaginal sling resulted in a dry perineum with few skin problems. Four patients required chronic suppressive oral antibiotic therapy while they had indwelling catheters.
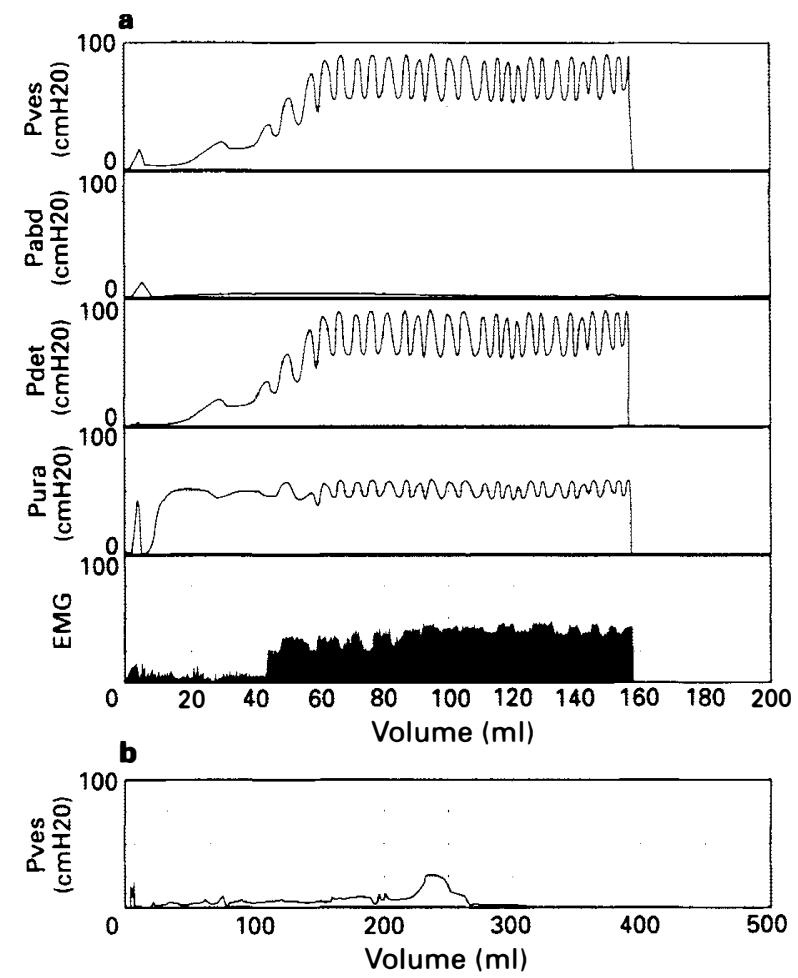

Figure 3 (a) Preoperative urodynamic evaluation demonstrated elevated intravesical pressure of $88 \mathrm{cmH}_{2} \mathrm{O}$ and detrusor-external sphincter dyssynergia. (b) Postoperative cystometry revealed low-pressure urinary drainage through the ileal bladder chimney and detrusor contraction generating $26 \mathrm{cmH}_{2} \mathrm{O}$ intravesical pressure. Pves: intravesical pressure $\left(\mathrm{cmH}_{2} \mathrm{O}\right)$; Pabd: intraabdominal pressure $\left(\mathrm{cmH}_{2} \mathrm{O}\right)$; Pdet: true detrusor pressure $\left(\mathrm{cmH}_{2} \mathrm{O}\right)$; Pura: urethral sphincter pressure; EMG: sphincter electromyography
This medication was stopped after the procedure and symptomatic urinary tract infection has not been evident.

Pelvic pain was a common complaint for the patients while they used an indwelling catheter for bladder management. After surgery, all eight patients with pain noted a resolution of their pelvic pain. Preoperatively, two spinal cord injury patients with lesions above T6 suffered with episodes of autonomic dysreflexia associated with catheter blockage or manipulation. Their symptoms of autonomic dysreflexia demonstrated significant improvement after surgery.

Intraoperatively, neither injury to a ureter nor an abdominal or pelvic organ occurred. In the immediate postoperative period, no fistulae, hemorrhage or sepsis developed. We have noted no loss of pubovaginal sling integrity, abdominal wall herniation, urethral destruction, bowel obstruction, metabolic disturbances, or urinary extravasation. Low-grade postoperative fever secondary to atelectasis or urinary tract infection occurred in nine patients. None of the patients developed postoperative sepsis. One patient with a bladder chimney procedure (patient 4 ) initially required nightly stomal catheterization to minimize residual urine volume (approximately $250 \mathrm{ml}$ ) for 6 months. This patient has had an otherwise uneventful postoperative course and has not required stomal catheterization for 3 years. Seven patients initially required anticholinergic medications for treatment of the sensation of urinary urgency, often referred to as bladder spasm, during the initial 3 postoperative months.

\section{Discussion}

The management of urinary incontinence in patients with advanced neurological disease demands a method that controls the continuous perineal wetting and avoids the risk of decubitus ulceration. In patients with adequate manual dexterity, vesical compliance and capacity, an intermittent catheterization program is the preferred treatment for neurogenic vesical dysfunction. ${ }^{10}$ McGuire and Savastano have thoroughly documented that neurogenic vesical dysfunction managed with intermittent catheterization minimizes the risks of urethral erosion, upper urinary tract injury, continual urinary incontinence, and febrile urinary tract infection which occur with indwelling catheter management. ${ }^{11}$

Indwelling catheters result in colonization of the urinary tract with microorganisms. The rate of urinary infection is approximately $5-7 \%$ for each day of catheterization. In fact, there is a $100 \%$ incidence of significant bacteriuria associated with long term indwelling catheters. ${ }^{12}$ The urinary tracts of patients with indwelling catheters are usually colonized by several organisms which may demonstrate multiple-drug resistance, particularly when broad-spectrum antibiotics are administered liberally over long periods of time. A small, fibrotic, low-compliance bladder can develop from the chronic infection and inflammation associated with an indwelling catheter. The small, contracted 
bladder can develop high pressure $\left(>40 \mathrm{cmH}_{2} \mathrm{O}\right)$ and thereby contribute to upper tract deterioration.

Indwelling catheters may be complicated by bladder calculi, serving as a nidus for calculus formation. Approximately $98 \%$ of the urinary tract calculi in patients with spinal cord injury, composed of calcium phosphate and magnesium ammonium phosphate, are secondary to urinary tract infection. Urinary stasis predisposes a patient to urinary tract infections, as well as to stone formation. Such stasis may occur from catheter kinking, malposition, or obstruction by urinary sediment.

The constant pressure of an indwelling urethral catheter may result in complete bladder neck and urethral destruction in the female, while in the male, traumatic hypospadias can result. The mechanism of injury from a chronically indwelling catheter may be pressure necrosis of the self-retaining balloon or spontaneous extrusions which occur during bladder spasm. In order to maintain a coaptive seal at the bladder neck, progressively larger diameter catheters are required as the urethra undergoes continual damage. Eventually, the continued erosion results in permanent urethral and sphincter destruction.

Indwelling catheters have been identified as a risk factor in the development of bladder cancer. Catheters cause chronic irritation and infection of the urothelium. Histological studies demonstrate a predominantly eosinophilic inflammatory response with urothelial dysplasia and squamous metaplasia noted at the site of the catheter. ${ }^{13}$ The exact significance of squamous metaplasia is unknown, but the incidence of these urothelial changes and bladder cancer is temporally related to the duration of the chronically indwelling catheter. A significant difference in the incidence of squamous metaplasia was noted between patients with spinal cord injury who were managed with indwelling catheters for more than 10 years $(80 \%)$ and those with a catheter less than 10 years $(42 \%)$ and those without a catheter $(20 \%)$. Squamous metaplasia is noted in patients who develop bladder cancer. However, it is not clear if there is progression from squamous metaplasia to frank carcinoma. ${ }^{14-15}$

The incidence of bladder cancer in patients with spinal cord injury has been reported to be $2-10 \%$, with the most common histological type being squamous cell carcinoma, either alone or with elements of transitional cell carcinoma. ${ }^{14-17}$ This incidence may be decreasing because of improvements in urological management and less dependence on indwelling catheters. ${ }^{18}$

Unfortunately, despite the increased efficacy and dramatically decreased morbidity of intermittent catheterization, most women are currently managed with an indwelling urethral catheter. A long term follow-up, of up to 15 years, in 108 women with neurogenic bladder dysfunction after spinal cord injury recently documented that 64.3 to $71 \%$ continue to be managed with an indwelling catheter. ${ }^{19}$ This method of management will eventually result in urethral destruction. Urinary drainage is best accomplished using a lowpressure reservoir with a catheterizable, continent stoma in those patients unable to self-catheterize the urethra. Patients with ongoing neurological dysfunction, however, such as those with a cervical spinal cord injury, may lack the dexterity or acuity to catheterize a stoma. Such patients may be best managed with the creation of an incontinent urostomy, which requires less attention and whose complication rate is lower than that of continent-type urinary diversions.

In patients with malignant bladder pathc!ogy, cystectomy is required in the course of establishing urinary diversion. Patients with neurogenic vesical dysfunction, however, need not be subjected to the rigors of cystectomy. In such cases, the bladder is best left in situ, even although urinary diversion is necessary to achieve perineal dryness and obviate the inconvenience and morbidity associated with urethral urinary incontinence. By leaving the bladder trigone undisturbed in the course of creating a urinary diversion with an ileal bladder chimney, ureteral integrity is not compromised. The risks of ureteral devascularization, urinary extravasation, and uretero-intestinal anastomotic disruption or stricture formation are thereby avoided entirely.

For those patients who have already suffered extensive damage to the continence mechanism, additional attention must be directed to the bladder neck and urethra. The surgical alternatives for a patulous, damaged, non-functional urethra include supravesical urinary diversion, transposition of the remaining urethra, surgical closure of the bladder neck, the placement of an artificial sphincter, or pubovaginal sling urethral compression. ${ }^{20-24}$ Surgical closure of the bladder neck has been reported to have an up to $25 \%$ chance of persistent fistulae. ${ }^{21}$ Implantation of the artificial urinary sphincter has been associated with urethral erosion and prosthesis infection. ${ }^{9}$

The pubovaginal sling has been reported mainly for the treatment of stress urinary incontinence secondary to intrinsic sphincter dysfunction. ${ }^{25-27}$ Chancellor et al reported the use of pubovaginal sling as a method of functional bladder neck closure in women with neurogenic vesical dysfunction. This method was chosen because of the documented incidence of postoperative fistula formation after a transabdominal or transvaginal bladder neck closure. Also highly significant, a functional closure avoids a completely closed urinary system. Pubovaginal sling offers a 'pop-off' safety valve which enables leakage, although at a high pressure, to occur. ${ }^{28}$

In conclusion, ileocystostomy with a cutaneous urostomy, the creation of a 'bladder chimney', is an alternative to suprapubic tube cystostomy or supravesical urinary diversion. Its creation is much less traumatic to the patient than formal cystectomy with intestinal conduit urinary drainage. The main advantage of the creation of a bladder chimney is that it avoids any need for a foreign body to aide in urinary drainage, such as a suprapubic tube or urethral catheter, while also not requiring manipulation of the ureters. In cases where sphincteric function has been compromised by a chronically indwelling catheter, the 
pubovaginal sling offers significant advantages over other forms of functional urethral closure. This potentially reversible surgery is applicable to most cases where urinary diversion is desired.

\section{References}

1 Thomas DG, Clarks SJ. The urological status of 86 females following spinal cord injury. Br J Urol 1979; 51: 515-517.

2 Barkin $\mathrm{M}$ et al. The urologic care of the spinal cord injury. J Urol 1983; 129: 335-339.

3 Lapides J, Diokno AC, Silber SJ, Lowe BS. Clean intermittent self-catheterization in the treatment of urinary tract disease. J Urol 1972; 107: 458-461.

4 Kuhn W, Rist M, Zaech GA. Intermittent urethral selfcatheterization: long term results (bacteriological evolution, continence, acceptance, complications). Paraplegia 1991; 29: 222-232.

5 McMaster WC, Nicholas JJ, Rosen JS. Intermittent catheterization for spinal-cord injury patients with chronic indwelling urethral catheters. Arch Phys Med Rehabil 1972; 53: 563-567.

6 Cordonnier JJ. Ileocystostomy for neurogenic bladder. J Urol 1957; 78: 605-610.

7 Blaivas JG, Jacobs BZ. Pubovaginal fascial sling for the treatment of complicated stress urinary incontinence. J Urol 1991; 145: $1214-1218$

8 McGuire EJ et al. Experience with pubovaginal slings for urinary incontinence at the University of Michigan. J Urol 1987; 138: 525-526.

9 Wan JL, McGuire EJ. Augmentation cystoplasty and closure of the urethra for the destroyed lower urinary tract. J Am Paraplegia Soc 1990; 13: 5-40.

10 Timoney AG, Shaw PJ. Urological outcome in female patients with spinal cord injury: the effectiveness of intermittent catheterization. Paraplegia 1990; 28: 556-5563.

$11 \mathrm{McGuire}$ EJ, Savastano JA. Comparative urological outcome in women with spinal cord injury. J Urol 1986; 135: 730-731.

12 Stover SL, Lloyd K, Waites KB, Jackson AB. Urinary tract infection in spinal cord injury. Arch Phys Med Rehabil 1989; 70: $47-54$
13 Goble NM, Clarke T, Hammonds JC. Histological changes in the urinary bladder secondary to urethral catheterization. $B r J$ Urol 1989; 63: 354-357.

14 Bejany DE, Lockhart JL, Rhamy RK. Malignant vesical tumors following spinal cord injury. J Urol 1987; 138: 1390-1392.

15 Bickel A, Culkin DJ, Wheeler JS. Bladder cancer in spinal cord injury patients. J Urol 1991; 146: 1240-1242.

16 Broecker BH, Klein FA, Hackler RH. Cancer of the bladder in spinal cord injury patients. J Urol 1981; 125: 196-197.

17 Melzak J. The incidence of bladder cancer in paraplegia. Paraplegia 1967; 4: 85-89.

18 Locke JR, Hill DE, Walzer Y. Incidence of squamous cell carcinoma in patients with long-term catheter drainage. $J$ Urol 1985; 133: 1034-1035.

19 Jackson AB, DeVivo M. Urological long-term follow-up in women with spinal cord injuries. Arch Phys Med Rehabil 1992; 73: $1029-1035$.

20 Mundy AR, Nurse DE, Dick JA, Murray KHA. Complex urinary undiversion. Br J Urol 1986; 58: 640-643.

21 Reid R, Schneider K, Fruchtman. Closure of the bladder neck in patients undergoing continent vesicostomy for urinary incontinence. J Urol 1978; 120: 40-42.

22 Feneley RCL. The management of female incontinence by suprapubic catheterization, with or without urethral closure. Br J Urol 1983; 55: 203-207.

23 Griffiths IH. Anterior transposition of the urethra. Br J Urol $1960 ; 32: 27-31$

24 Zimmern PE, Hadley HR, Leach GE, Raz S. Transvaginal closure of the bladder neck and placement of a suprapubic catheter for destroyed urethra after long-term indwelling catheterization. J Urol 1985; 134: 554-557.

25 Blaivas JG, Olsson CA. Stress incontinence: classification and surgical approach. J Urol 1988; 139: 727-731.

26 Morgan JE, Farrow GA, Stewart RN. The Marlex sling operation for the treatment of recurrent stress urinary incontinence: a 16-year review. Am J Obstet Gynecol 1985; 151: 224-226.

$27 \mathrm{Raz} \mathrm{S}$ et al. Fascial sling to correct male neurogenic sphincter incompetence: The McGuire/Raz approach. J Urol 1988; 139: $528-531$.

28 Chancellor MB, Erhard MJ, Kiilholma PJ, Rivas DA. Functional urethral closure with pubovaginal sling for destroyed female urethra after long-term urethral catheterization. Urology 1994; 43: 499-505. 